\title{
P-ANCA senza positività per anticorpi anti-mieloperossidasi (ANTI-MPO): associazioni cliniche
}

\author{
G. Gregorini, P. Tira, R. Gorla ${ }^{1}$, E. Prati ${ }^{1}$ \\ Divisione di Nefrologia e Servizio di ${ }^{1}$ Immunologia Clinica, Spedali Civili di Brescia
}

1 test in Immunofluorescenza Indiretta (IFI) su neutrofili normali fissati in alcool è il test più largamente usato per la ricerca di ANCA. Possono emergere due pattern di positività: il pattern citoplasmatico (C-ANCA) in cui la fluorescenza si presenta a grosse aree nel citoplasma, od il pattern di tipo perinucleare (P-ANCA) in cui la fluorescenza si distribuisce intorno al nucleo e sul nucleo. Il pattern C-ANCA è dovuto nella quasi totalità dei casi alla presenza di anticorpi anti-Proteinasi 3 (anti-PR3): la positività del test in IFI di per sé ha un valore diagnostico elevato, è in pratica un marker di Granulomatosi di Wegener e di Micropoliangite (1). Il pattern tipo $\mathrm{P}$ - ANCA è nel $50 \%$ dei casi dovuto ad anticorpi anti-mieloperossidasi (anti-MPO): in questi casi è un marker diagnostico di Micropoliangite e soprattutto della sua variante di vasculite limitata al rene (2).

Il valore di un test positivo con pattern P-ANCA senza presenza in ELISA di anti-MPO, per la diagnosi di vasculite, è molto limitato a causa di due fatti: 1) L'interferenza nel test degli anticorpi antinucleo (ANA): la presenza di ANA dà un pattern tipo P-ANCA nel preparato di neutrofili. Quindi la presenza nel siero di ANA rende difficoltosa la distinzione tra ANA e P-ANCA.

2) La presenza di P-ANCA senza anti MPO in malattie diverse dalle vasculiti: nell'artrite reumatoide, nelle malattie in- fiammatorie intestinali e nelle malattie epatiche autoimmuni ed in alcune epatopatie croniche sono stati trovati, da vari autori, in varie percentuali di pazienti, PANCA senza anti-MPO. I determinanti antigenici verso cui sono diretti i P-AN$\mathrm{CA}$, in queste malattie, non sono tuttora definiti $(3,4)$.

\section{Distinzione tra ANA e P-ANCA}

Nella pratica clinica questo problema si pone frequentemente: prima di tutto perché i pazienti affetti da vasculite condividono buona parte della sintomatologia con i pazienti affetti da altre malattie del connettivo in cui sono quasi costantemente presenti gli ANA e quindi non è raro che questi pazienti siano sottoposti alla ricerca di ANCA. Inoltre, come in altre malattie autoimmuni, anche nelle vasculiti sistemiche è frequente la contemporanea presenza di ANA. Le modalità per distinguere i due tipi di autoanticorpi sono elencate nella Tabella I.

\section{TAB. I - LA PRESENZA NEL SIERO DI ANTICORPI ANTINUCLEO (ANA) DÀ UN PATTERN P-ANCA NEL PREPARATO DI GRANULOCITI NEU- TROFILI}

\section{Come distinguere fra ANCA e ANA?}

1) Cercare nel citocentrifugato se risultano positivi anche i nuclei dei linfociti, si conferma in questo modo che si tratta di ANA

2) Confermare la presenza di ANA sulle cellule HEP-2

3) Test ELISA per antigeni dei neutrofili (MPO, elastasi, ecc.)

4) Fissare il preparato di granulociti neutrofili con formaldeide ->

- a differenza della fissazione in alcool non fà fuoriuscire dai granuli gli antigeni contenuti nei granuli del citoplasma (es MPO) e non ne provoca la dislocazione in torno e sul nucleo

- distrugge la gran parte dei determinanti antigenici nucleari ed impedisce quindi la evidenziazione degli ANA:

a) con la tecnica usuale della microscopia in fluorescenza

b) con l'analisi citofluorimetrica 
Utilizzo di una metodica citofluorimetrica per la distinzione tra ANCA e ANA

Partendo da una metodica citofluorimetrica per la ricerca degli ANCA recentemente validata, il nostro gruppo ha, tra gli altri aspetti, considerato anche la possibile applicazione di questa metodica per differenziare ANCA e ANA.

A questo scopo la metodica è stata modificata essenzialmente usando una diversa tecnica di fissazione dei neutrofili.

La fissazione dei globuli bianchi con una soluzione di Paraformaldeide al 19\% per $15^{\prime}$ a $4^{\circ} \mathrm{C}$ sembra permettere il legame fra gli antigeni contenuti nel citoplasma e gli ANCA presenti nel siero, mentre non permette il legame fra gli antigeni contenuti nel nucleo e gli ANA. Non è tuttora stabilito se questo dipenda da una selettiva distruzione dei determinanti an-

TAB. II - SCHEMA DELLO STUDIO

Tutti i sieri con positività P-ANCA

$(+->++++)$ con test ELISA per anticorpi anti-Mieloperossidasi negativo testati nel periodo giugno 1987 -> gennaio 1995 presso gli Spedali Civili di Brescia

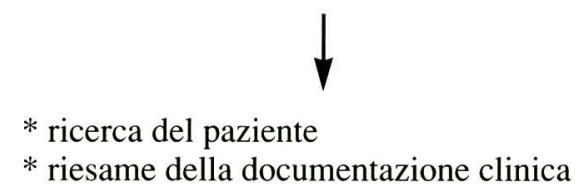

* riesame della documentazione clinica

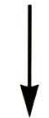

92 pazienti, 78 rintracciati $(85 \%)$

distribuzione in rapporto alla presenza di ANA

ANA non testati:

ANA positivi:

ANA negativi:
$14 \mathrm{pz}$ (6 non rintracciati)

$42 \mathrm{pz}$ (6 non rintracciati)

$36 \mathrm{pz}$ (2 non rintracciati)

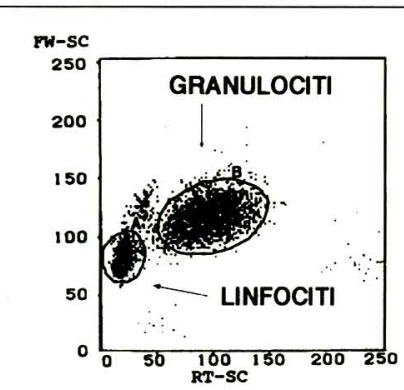

Citogramma generatore

L'analisi permette l'individuazione contemporanea

delle regioni linfocitaria (A) e granulocitaria (B)

ISTOGRAMMI DI FLUORESCENZA

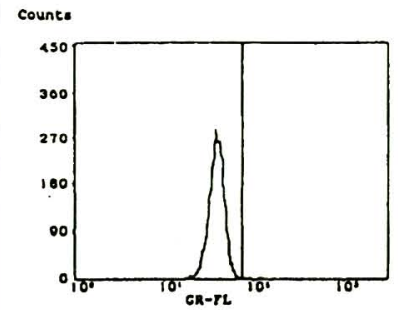

Soggetto sano

Non rilevazione di fluorescenza in entrambe le popolazioni cellulari

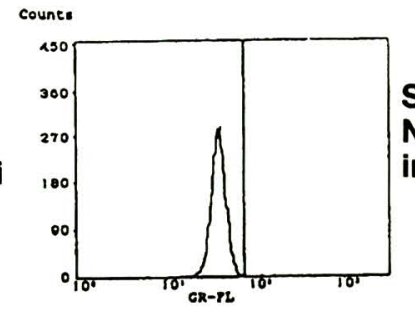

Soggetto ANA positivo Non rilevazione di fluorescenza in entrambe le popolazioni cellulari
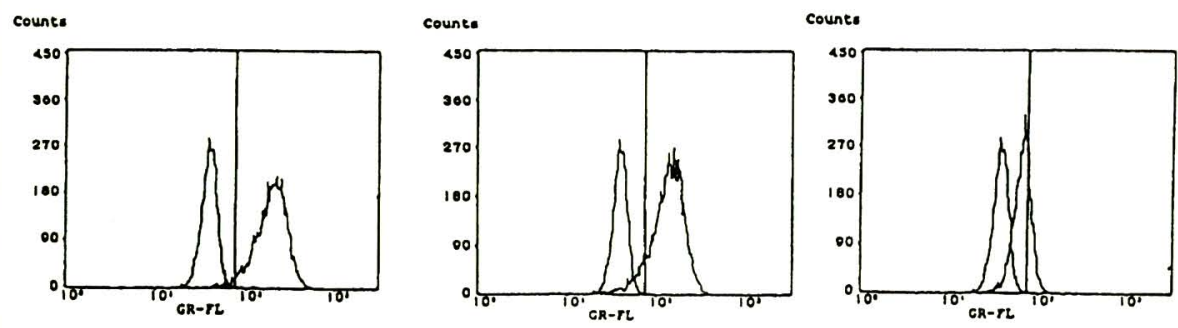

Soggetti ANCA positivi Rilevazione di fluorescenza nella sola popolazione granulocitaria Si individua una diverasa intensità di fluorescenza fra i diversi soggetti

Fig. 1 - Analisi citofluorimetrica per la rilevazione di anticorpi anticitoplasma dei granulociti 
tigenici nucleari o se dipenda dall'effetto di questa fissazione sulla membrana del nucleo che la renderebbe impermeabile all'anticorpo presente nel siero da testare. Come risulta dalla Figura 1, che mostra i dati preliminari del nostro lavoro:

1) I soggetti sani senza autoanticorpi si collocano nella regione che viene delimitata come regione negativa.

2) I soggetti noti ANA positivi non mostrano fluorescenza né sui granulociti, né sui linfociti e si comportano quindi come i soggetti sani

3) I soggetti noti ANCA positivi, mostrano la presenza di fluorescenza a livello dei granulociti con linfociti negativi.

4) L'intensità del picco di fluorescenza dei diversi soggetti ANCA positivi si dimostra variabile sul canale medio e ciò sembra indicare una correlazione tra picco di fluorescenza e titolo di autoanticorpi.

\section{Scopo dello studio}

Lo studio è stato finalizzato a:

- valutare le associazioni cliniche di un test P-ANCA positivo senza positività della ricerca degli anti-MPO

- valutare soprattutto se e con quale frequenza un test P-ANCA senza positività per anti-MPO è associato a vasculite sistemica.

TAB. III - P-ANCA CON RICERCA DEGLI ANTI-MPO NEGATIVA: PAZIENTI CON ANA TEST POSITIVO

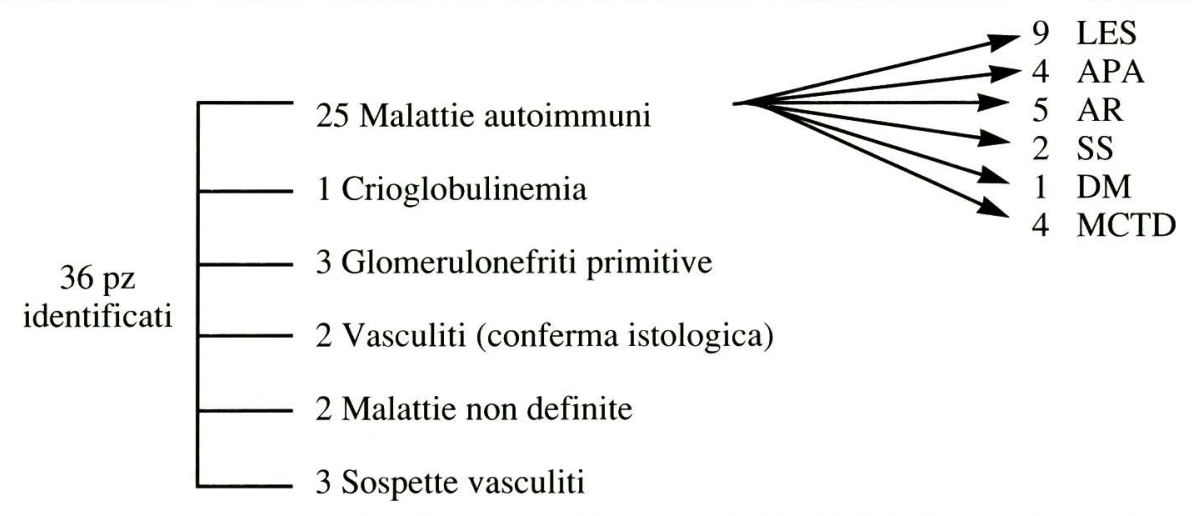

TAB. IV

B.A. $0^{\prime} 60$ anni

muratore dall'età di 14 anni

1993/5 Febbricola, astenia, artralgie, sinusite mascellare sx es. urine neg, RA test e WR neg, PCR pos

breve ciclo di trattamento con steroidi

1994/2 Intervento di by - pass aorto - coronarico. Nel post - operatorio:

febbre elevata, alterazioni urinarie, P-ANCA +++

1994/6 Ricovero in nefrologia: alterazioni urinarie minime, test di flogosi neg

IFI: P-ANCA +++ (ANA+). Biopsia renale: 6/12 glomeruli ialini, nei restanti aree sclerosi focale e fibrosi periglomerulari. IFI: IgM 1+ focale segmentale, C3 1+idem

L.G. o 56 anni

per 25 anni addetto alla costruzione di stampi per acciaierie (utilizzo di sabbia silicica,

lana di vetro e resine)

1991: $\quad$ Riscontro di silicosi

1992: Valutato nell'ambito dello studio "ANCA/SILICOSI":

$\mathrm{Hb}$ urine tracce, ANCA neg, test di flogosi neg

1993/4: Febbre, calo ponderale, artromialgie, pericardite alt. urinarie.

P-ANCA +++ (ANA+)

Biopsia renale: aumento cellularità e matrice mesangiale. IFI: negativa

TAB. V - P-ANCA CON RICERCA DEGLI ANTI-MPO NEGATIVA: PAZIENTI CON ANA TEST NEGATIVO

$\begin{array}{cl}\text { Miscellanea } \\ 5 & \text { Artrite reumatoide } \\ 3 & \text { Colite ulcerosa } \\ 1 & \text { Epatocolangite sclerosante } \\ 1 & \text { Artrite psoriasica } \\ 1 & \text { Crioglobulinemia } \\ 3 & \text { Nefropatie (non vasculiti) } \\ 2 & \text { Malattie non definite }\end{array}$
Neoplasie
1 Mielofibrosi
1 Carcinoma surrenalico
(metastatizzato)
1 Linfoma di Hodgking
Infezioni
1 Infezione da HIV
1 Sepsi da Staphylococcus aureus
Malattie Polmonari
1 Malattia di Rendu-Osler con emorragia polmonare
1 Fibrosi polmonare di incerta etiologia

$11 \quad$ vasculiti

\section{Metodi}

Sono stati retrospettivamente esaminati i risultati relativi ai sieri testati dal nostro gruppo a partire dal momento in cui la metodica di ricerca di ANCA è stata applicata estensivamente (giugno 1987) fino al gennaio 1995. Sono stati selezionati tutti i sieri che risultavano positivi al test in IFI con pattern P-ANCA e che risultavano negativi al test ELISA per antiMPO. Sono stati identificati i pazienti e riesaminata la documentazione clinica.

Al momento in cui lo studio è stato intrapreso un buon numero di questi sieri non era più disponibile e non potevano quindi essere ritestati secondo la nuova metodica che consente di distinguere i P-ANCA dagli ANA.

Per questo, non potendo eliminare la possibile interferenza sulla positività del test derivante dalla presenza di ANA, i pazienti sono stati prima di tutto separati in 3 gruppi ANA positivi, ANA negativi e pazienti non testati per gli ANA (vedi Tab. II). 
TAB. VI - P-ANCA CON RICERCA DEGLI ANTI-MPO NEGATIVA: PAZIENTI CON ANA TEST NEGATIVO

\section{Pazienti con vasculite}

2 pz con processo ulcero - necrotico a carico della mucosa nasale con distribuzione del setto (tutti e due) e deformazione a sella del dorso del naso (1 pz). Biopsia della mucosa nasale compatibile con Granulomatosi di Wegener (1 pz).

$1 \mathrm{pz}$ con sarcoidosi, ulcere a carico della mucosa orale e nasale, numerosi noduli sottocutanei, porpora palpabile e sintomi sistemici (vasculite confermata istologicamente a livello della mucosa orale e nasale, nei noduli sottocutanei e nelle lesioni cutanee)

4 pz con micropolingite e Glomerulonefrite extracapillare necrotizzante paucimmune

2 pz con sintomi sistemici e glomerulonefrite rapidamente progressiva (RPGN) senza conferma istologica

2 pz con IRC avanzata e: $\quad 1$ pz + episodio di emorragia polmonare + dermatite cronica psoriasiforme

$1 \mathrm{pz}+$ ipertiroidismo, multineurite, bronchiectasie

In tutti i pazienti gli ANCA sono stati testati in IFI e con test ELISA. Il test in IFI veniva eseguito secondo la metodica delineata al $1^{\circ}$ Workshop Internazionale sugli ANCA (5), i test in ELISA venivano effettuati usando come substrato sia un estratto acido di citoplasma dei neutrofili come fonte non purificata di PR3, sia i Mieloperossidasi Umana purificata (Calbiochem). Gli ANA venivano valutati con la tecnica di IFI su cellule HEP2 usando il siero ad una diluizione di 1:80.

\section{Risultati}

92 pazienti presentavano una positività P-ANCA senza anti-MPO. Dei 78 pazienti identificati $85 \%$ del numero totale) veniva riesaminata la documentazione clinica. In circa il 50\% dei pazienti risultavano presenti gli ANA (Tab. II). Come era prevedibile circa $3 / 4$ dei pazienti con positività degli ANA erano affetti da un'altra malattia autoimmune ed è molto verosimile che in questi pazienti

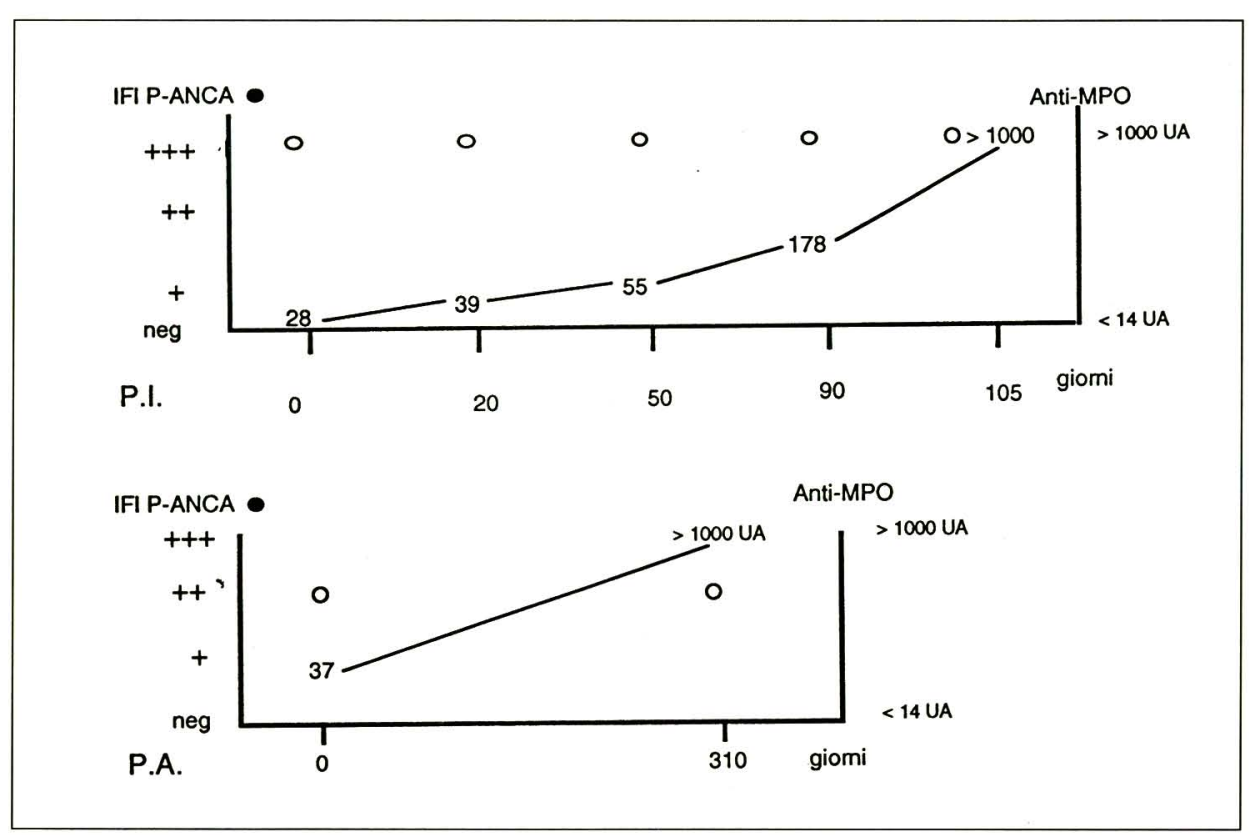

Fig. 2 - Esempi di andamento dissociato nel tempo della positività del Test in IFI e del Test in ELISA per anti - MPO la positività P-ANCA fosse dovuta alla inteferenza degli ANA (Tab. III). È interessante notare come in questo gruppo di pazienti ANA positivi ci fossero 5 pazienti in cui la diagnosi era di sicura o sospetta vasculite. Si trattava di un caso presentatosi con un quadro di multineurite in cui la biopsia del nervo era compatibile con vasculite, di un caso di Glomerulonefrite ed ulcere cutanee con biopsia della cute dimostrante vasculite leucocitoclasica, un caso di polineurite sensitivo - motoria, fibrosi retroperitoneale e fibrosi polmonare e di due casi le cui caratteristiche cliniche sono esposte nella Tabella IV.

La Tabella V illustra le diagnosi dei pazienti con P-ANCA con anticorpi antiMPO negativi e con anticorpi antinucleo negativi, quindi di pazienti con sicuri PANCA.

Nove pazienti presentavano una delle patologie non vasculitiche in cui è descritta la presenza di P-ANCA non anti-MPO: artrite reumatoide, malattie infiammatorie intestinali ed epatopatie autoimmuni. Considerando l'intero gruppo di pazienti, indipendentemente dalla suddivisione secondo la presenza degli ANA, 10 pazienti erano affetti da artrite reumatoide, 7 erano affetti da malattie infiammatorie intestinali ed un solo paziente era affetto da epatopatia autoimmune (epatocolangite sclerosante).

Nel gruppo di pazienti con ANA negativi emergevano anche alcuni tipi di malattie in cui è stata descritta la presenza di "false positività" degli ANCA al test in IFI sia con pattern P-ANCA che con pattern C-ANCA. Si trattava di infezioni, l'infezione da HIV e la sepsi da Gram positivi, malattie neoplastiche (linfomi e carcinomi) e malattie polmonari croniche $(6,7)$. Undici pazienti in questo gruppo presentavano vasculiti (Tab. VI). Di questi, 8 pazienti presentavano vasculiti con le caratteristiche di quelle tipicamente associate ad anti-MPO: Micropoliangiti e forme limitate al rene.

In due casi, infine, la positività in fluorescenza era dissociata nel tempo dalla positività del test in ELISA per gli antiMPO. Questa osservazione era già stata fatta in alcuni pazienti nel corso del trattamento immunosoppressivo: il test in immunofluorescenza mostrava una persistente positività mentre il titolo degli anticorpi nel test in ELISA era normalizzato. La Figura 2 mostra l'andamento del test nei due pazienti: a fronte di un test 
nettamente positivo in immunofluorescenza vi era un titolo del test di antiMPO in ELISA molto vicino al limite superiore allo norma. I pazienti venivano tenuti in osservazione e monitorati nel tempo e si assisteva ad un netto incremento del titolo degli anti-MPO. È da segnalare che in entrambi i casi la malattia era clinicamente evidente alla prima osservazione.

\section{Discussione e conclusioni}

La presenza di un test positivo alla IFI con pattern P-ANCA con una ricerca negativa di anticorpi anti-MPO, non può né confermare né escludere la presenza di una vasculite. La presenza di ANA esercita un'importante interferenza nel test: in pratica è molto difficile distinguere su di un preparato di granulociti neutrofili i P-ANCA dagli ANA.

È quindi sempre molto importante conoscere nel singolo paziente se vi è la presenza di ANA. In buona parte dei casi la positività $\mathrm{P}$-ANCA è solo dovuta all'interferenza degli ANA. D'altra parte come in tutte le malattie autoimmuni anche nelle vasculiti è molto frequente la coesistenza di più tipi di autoanticorpi (la ricerca di ANA è positiva nel $13 \%$ dei nostri casi di vasculite con positività al test per gli anti-PR3 ed anti-MPO). La situazione di coesistenza di ANA e P-ANCA può essere solo chiarita ricorrendo alla fissazione dei granulociti con formaldeide come descritto nella Figura 1.

Anche la nostra esperienza conferma la presenza di P-ANCA senza anti-MPO nell'artrite reumatoide, nelle malattie infiammatorie intestinali e nella epatocolangite sclerosante, tuttavia questo gruppo costituisce solo il $23 \%$ dei casi nella nostra casistica.

Una positività P-ANCA senza anti-MPO può essere trovata in corso di infezioni (HIV, sepsi da GRAM+), in corso di neoplasie (linfomi) ed in corso di malattie polmonari croniche apparentemente primitive. Queste possono essere considerate delle vere e proprie false positività che spesso (come nel caso delle infezioni) scompaiono con la remissione della malattia.

E importante rilevare che quadri di vasculite sistemica non dimissibili da quelli che si accompagnano alla positività degli anticorpi anti-MPO possono presentarsi con una positività del solo test in immunofluorescenza con pattern P-ANCA. Un reperto simile è stato descritto da altri autori e solo in alcuni casi sono stati identificati specificità antigeniche diverse dalla mieloperossidasi (es. l'elastasi, lattoferrina) $(8,9)$.

Questi pazienti non sembrano presentare caratteristiche cliniche diverse dagli altri e simili tra di loro al punto da poter configurare un gruppo a sé stante.

Va infine segnalata la possibilità di una dissociazione temporanea fra test in IFI (che resta positivo) e test in ELISA per anti-MPO, che si negativizza.

Queste osservazioni devono indurre, in condizioni cliniche suggestive, a dare rilievo ad un test con queste caratteristiche e devono indurre ad espletare le indagini per confermare la diagnosi di vasculite.

\section{BIBLIOGRAFIA}

1. Van der Woude FJ, Rasmussen N, Lobatto S, et al. Autoantibodies against neutrophils and monocytes: a new tool for diagnosis and marker of disease activity in Wegener's Granulomatosis. Lancet 1985; i: 425-9.

2. Falk RJ, Jennette JC. Antineutrophil cytoplasmic autoantibodies with specificity for myeloperoxidase in patients with systemic vasculitis and idiopathic necrotizing and crescentic glomerulonephritis. New Engl J Med 1988; 318: 1651-7.

3. Wiik A. Granulocyte-specific antinuclear antibodies. Possible significance for the pathogenesis, clinical features and diagnosis of rheumatoid arthritis. Allergy 1980; 35: 263-89.

4. Snook JA, Chapman RW, Fleming K, Jewell DP. Anti-neutrophil neuclear antibody in ulcerative coliti, Crohon's disease and primary sclerisis cholangitis. Clin Exp Immunol 1989; 76: 30-3.
5. Wiik A. Delineation of a standard procedure for indirect immunofluorescence detection of ANCA. Acta Pathol Microbiol Immunol Scand 1989; 97: 12-3.

6. Koderisch J, Andrassy K, Rasmussen N, Hartmann M, Tilgen W. False-positive anti-neuthil cytoplasmic antibodies in HIV infection. Lancet 1990; 335: 1227-8.

7.

Nieuwhof C, Theunissen $\mathrm{R}$, Goldschmeding R, van Breda Vriesman PJC. Wegener granuloma like renal lesion and ANCA in subacute bacterial endocarditis. (Abstr.) XXIX $^{\text {th }}$ Congress of European Dialysis and Transplant Association-European Renal Association Paris 1992; 77.

8. Savige JA, Gallicchio $M$, Georgiou T, Davies DJ. Diverse target antigens recognized by circulating antibodies in anti-neutrophil cytoplasm antibody-associated renal vasculitides. Clin Exp Immunol 1990; 82: 238-43.

9. Lesavre P, Noel LH, Gayno S, et al. Atypical autoantigen target of perinuclear antineutrophil cytoplasm antibodies (P-ANCA); specificy and clinical associations. Journal of Autoimmunity 1993; 6: 185-95. 